> Les modifications post-traductionnelles de protéines sont essentielles à la régulation de leur fonction et, par conséquent, au maintien de l'intégrité cellulaire. Ces modifications interviennent à tous les niveaux de la communication entre les neurones du système nerveux central. La sumoylation est une modification post-traductionnelle qui crée une liaison covalente entre une lysine de la protéine cible et une petite protéine appelée SUMO. C'est un processus réversible qui fait intervenir des enzymes spécifiques. La sumoylation est un processus régulateur fonctionnel bien connu de l'activité nucléaire et il est maintenant clairement établi que dans les neurones, il assure également un rôle majeur pour de nombreux processus extranucléaires. De plus, la sumoylation intervient dans la transmission synaptique et a été impliquée récemment dans plusieurs désordres neurologiques. <

\section{Nouvelles fonctions extranucléaires de Ia sumoylation des protéines dans le système nerveux central}

\section{Stéphane Martin}

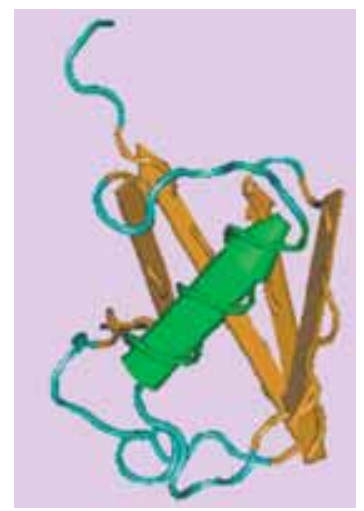

Institut de neuromédecine moléculaire, Institut de pharmacologie moléculaire et cellulaire, Centre national de la recherche scientifique, UMR 6097, Université de Nice Sophia-Antipolis, 660 , route des Lucioles, Sophia-Antipolis, 06560 Valbonne, France. smartin@in2m.cnrs.fr
La modification post-traductionnelle des protéines est un processus enzymatique qui consiste en l'ajout de groupements chimiques, de sucres, de lipides ou encore de polypeptides après la synthèse protéique. Ces modifications peuvent altérer la conformation des protéines, influer sur leur localisation cellulaire, dicter leur stabilité ou encore agir sur leur activité et donc sur leur fonction physiologique. Par exemple, les processus synaptiques de la communication neuronale sont particulièrement complexes et finement régulés, en partie grâce à ces modifications post-traductionnelles. La sumoylation est une modification post-traductionnelle dont les fonctions en dehors du noyau sont peu connues. Cette synthèse fait le point sur notre connaissance des fonctions extranucléaires de la sumoylation dans le système nerveux central et discute leur implication dans les maladies neurodégénératives.

\section{Qu'est-ce que la sumoylation?}

La sumoylation a été découverte en 1996 [1]. C'est un processus enzymatique qui consiste en l'attachement covalent d'une protéine appelée SUMO (small ubiquitinlike modifier) sur la lysine de la séquence consensus $\Psi \mathrm{KxD} / \varepsilon$ d'une protéine cible ( $\Psi$, acide aminé hydrophobe ; $K$, lysine modifiée; $x$, résidu quelconque et $D / \varepsilon$, aspartate ou glutamate). Il faut cependant noter que la littérature rapporte un nombre de plus en plus élevé de protéines sumoylées sur des lysines n'appartenant pas à un site consensus de sumoylation. Bien que la séquence primaire de SUMO soit différente de celle de l'ubiquitine, la structure tridimensionnelle de ces deux protéines est similaire (Figure 1). La sumoylation est un processus essentiel puisque l'invalidation des enzymes de la sumoylation est délétère pour les cellules eucaryotes [2, 3].

Le terme SUMO regroupe une famille de 4 protéines (SUM01-4), formées chacune d'environ 100 résidus. Les formes SUM01-3 sont exprimées dans le cerveau alors que l'expression de SUMO-4 semble restreinte aux cellules des reins, de la rate et des ganglions lymphatiques. SUMO-2 et SUMO-3 ne diffèrent que par 3 résidus et ne présentent que $47 \%$ d'homologie avec SUMO-1. De manière très intéressante, SUMO-2 et 3 peuvent former des chaînes de poly-SUMO, contrairement à SUMO-1 qui ne comporte pas le résidu de lysine indispensable à la formation de ces chaînes [4]. 


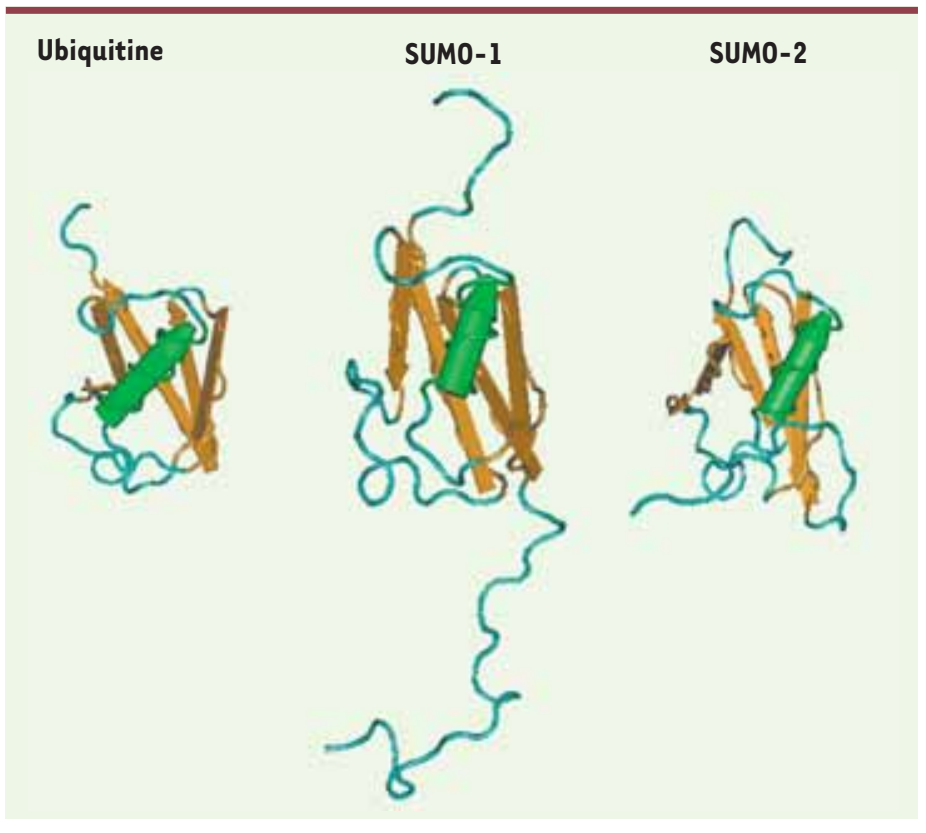

Quel est le mécanisme enzymatique de la sumoylation?

SUM0 est synthétisée sous forme de précurseurs inactifs (Figure 2). La maturation se fait par l'activité hydrolase d'enzymes spécifiques appelées SENP (SUMO/Sentrin-specific protease). Ces enzymes permettent l'exposition d'un doublet de glycine au niveau carboxy-terminal de SUMO où se fera la liaison avec le résidu lysine de la protéine cible. La forme mature SUMO-GG est activée par le complexe $\varepsilon l$ de manière dépendante de l'ATP. La forme activée se lie alors à l'enzyme Ubc9 qui a la particularité d'être l'unique enzyme de conjugaison de la voie de sumoylation [5]. Ubc9, avec ou sans l'aide d'une SUMO \&3, catalyse la liaison covalente de SUMO sur la lysine de la protéine cible (Figure 2).

La sumoylation est un processus réversible très dynamique. La désumoylation est catalysée par l'activité isopeptidase des enzymes SENP (Figure 2). II existe 6 isoformes de ces enzymes chez les mammifères (SENP1-7; les séquences SENP3 et SENP4 sont identiques; pour revue [6]). Ces enzymes présentent des distributions subcellulaires distinctes. En effet, alors que SENP2-3 sont nucléaires, SENP1 est exprimé à la fois dans le noyau et le cytoplasme. SENP5 est également présent dans le noyau mais aussi dans les mitochondries. SENP6 est majoritairement cytoplasmique et localisé exclusivement dans le cerveau et les organes reproducteurs [6].

\section{Quelles sont les fonctions cellulaires de la sumoylation?}

Le rôle nucléaire de la sumoylation est le mieux caractérisé avec des fonctions essentielles dans la réparation de l'ADN, la régulation de la transcription ou le transport des protéines à travers la membrane nucléaire (pour revue $[7,8]$ ). Depuis peu, des études rendent compte de fonctions non nucléaires de la sumoylation. SUMO peut intervenir dans la voie de dégradation des protéines en agissant positivement ou négativement sur le processus de l'ubiquitinylation. SUMO peut empêcher la dégradation des protéines en entrant en compétition avec
Figure 1. Structure tridimensionnelle de l'ubiquitine et de SUMO1 et SUM02. Représentation schématique de la structure des formes humaines de l'ubiquitine et de SUMOl et SUMO2. SUMO2 et SUMO3 ne diffèrent que par 3 acides aminés, leur structure est quasi-identique. II est important de noter que malgré une homologie de séquence primaire minimale, les structures tridimensionnelles de l'ubiquitine et des paralogues de SUMO sont très semblables.

I'ubiquitine pour la modification de lysines initialement ciblées par les enzymes de la voie de l'ubiquitinylation ou bien favoriser la dégradation comme c'est le cas lors de la reconnaissance de protéines sumoylées par des ubiquitines ligases [9].

La régulation de la fonction des protéines par la sumoylation a atteint un niveau de complexité supplémentaire du fait de la possibilité d'interactions protéiques non covalentes avec SUMO. Plus spécifiquement, il a été rapporté que de nombreuses protéines contenaient des motifs spécifiques d'interaction avec SUMO appelés domaine SIM (SUMO-interacting motif). Ainsi, la sumoylation peut moduler les interactions protéiques soit directement, en modifiant de façon covalente l'un des partenaires d'interaction, soit indirectement, en s'associant de façon non covalente avec SUMO via les domaines SIM [10]. Cependant, bien que des motifs SIM aient été identifiés sur de nombreuses protéines extranucléaires, aucune interaction non covalente de ce type en dehors du noyau n'a encore été rapportée.

Bien qu'il y ait peu de données concernant les cibles et les fonctions de la sumoylation en dehors du noyau, cet axe de recherche est particulièrement actif [11]. J'ai principalement focalisé cette synthèse sur le rôle fonctionnel des substrats neuronaux extranucléaires connus de la sumoylation (Figure 3).

\section{Rôles cytoplasmiques de la sumoylation}

\section{Régulation de la phosphorylation}

Le processus de phosphorylation fait partie des cascades de signalisation cellulaire et peut être modulé par la sumoylation. Par exemple, la protéine kinase FAK (focal adhesion kinase) est un substrat de la sumoylation. FAK régule la motilité des cônes de croissance et son action est déterminante pour les interactions entre matrice extracellulaire et cytosquelette. La sumoylation de FAK induit son autophosphorylation. Cette étape est cruciale pour la liaison de FAK avec la kinase Src qui, à son tour, phosphoryle FAK pour l'activer totalement [12]. 


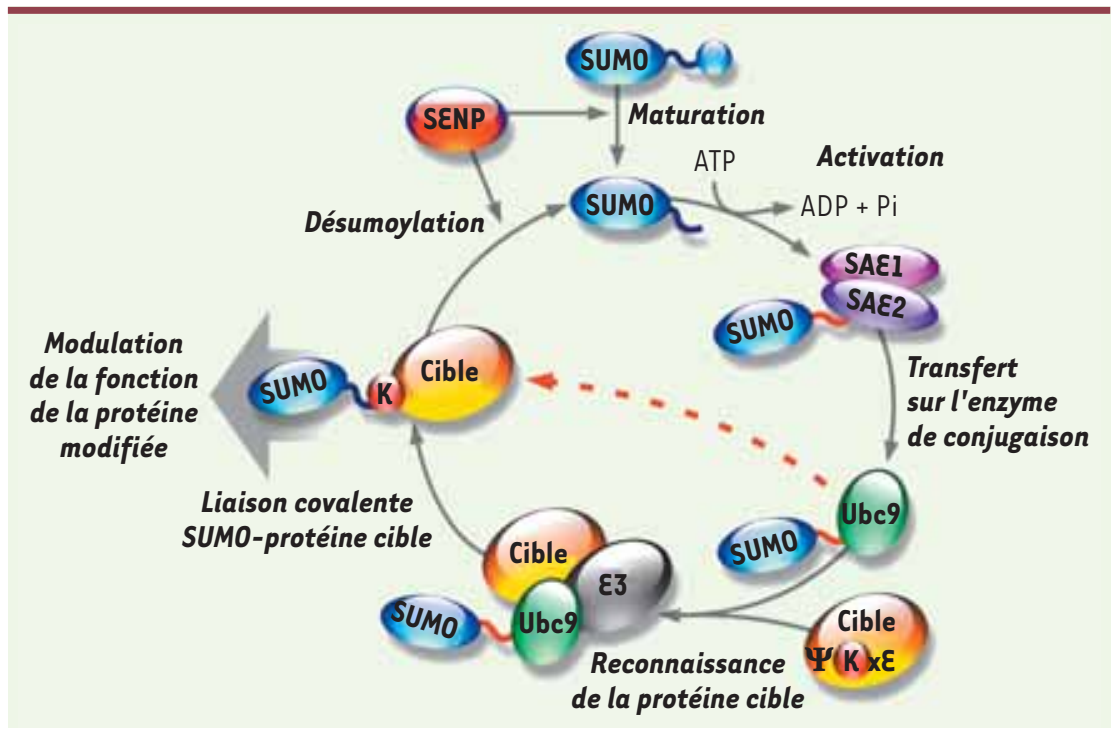

Figure 2. La voie enzymatique de sumoylation/ désumoylation. Les paralogues de SUMO sont synthétisés sous forme de précurseurs inactifs et sont maturés par l'activité hydrolase des enzymes spécifiques de désumoylation SENP. SUMO est ensuite activé par le complexe $\varepsilon l$ qui est formé des enzymes SAEl et SAE2 (également appelées respectivement Aosl et Uba2). Cette liaison covalente entre SUMO et SAE2 est un processus utilisant de I'ATP. SUMO est alors transférée sur la cystéine du site actif de l'enzyme de conjugaison Ubc9. Ubc9 peut soit catalyser directement le transfert de SUMO sur le substrat à modifier (flèche en pointillés), soit transférer SUMO sur la protéine cible via un complexe avec une enzyme ligase $(\varepsilon 3)$. Dans les deux cas, une liaison isopeptidique se forme entre SUMO et la lysine de la séquence consensus $\Psi$ KxD/E de la protéine cible où $\Psi$ représente un acide aminé hydrophobe ; K, la lysine cible; $x$, un résidu quelconque et $D / \varepsilon$, un aspartate ou un glutamate. SUMO peut ensuite être dynamiquement enlevé des protéines cibles sous l'effet de l'activité isopeptidase des enzymes de désumoylation SENP.

PTPIB (protéine tyrosine phosphatase 1B) est une phosphatase exprimée principalement dans l'hypothalamus où elle participe à la régulation de la signalisation de la leptine, une hormone de satiété [35]. Elle est en outre impliquée dans la régulation de la signalisation de l'insuline via son activité de phosphatase sur de nombreux récepteurs à activité tyrosine kinase. La sumoylation de PTPIB en réponse à l'insuline diminue à la fois l'activité et l'expression de PTP1B [13]. Ce mécanisme pourrait participer au contrôle neuronal de la prise alimentaire.

\section{Régulation fonctionnelle des mitochondries}

L'élévation de l'activité neuronale est suivie de l'augmentation du nombre de mitochondries dans les épines dendritiques. DRP-l (dynamin-related protein), un analogue mitochondrial de la dynamine, régule le nombre de mitochondries en participant à la fission de ces dernières. $B A X$, une protéine pro-apoptotique, induit la sumoylation de DRP-1, ce qui la protège de la dégradation [14, 15]. L'enzyme de désumoylation SENP5 est également importante pour la fission et le maintien de la morphologie mitochondriale [16]. Ces données, auxquelles s'ajoute l'identification de multiples substrats de la sumoylation dans les mitochondries cérébrales [17], laissent entrevoir un rôle prépondérant de cette modification dans l'homéostasie mitochondriale et le processus d'apoptose.

\section{Régulation de la signalisation par les protéines $G$}

RGSZI (regulator of $G$ protein signaling Z1) et RGSZ2 sont des GTPases exprimées exclusivement dans le cerveau et qui permettent la régulation de la sous-unité $G \alpha$ des protéines G. RGSZ1 et RGSZ2 sont impliquées dans la désensibilisation des récepteurs aux opiacés de type $\mu$ qui appartiennent à la famille des récepteurs couplés aux protéines $G(R C P G)$. Dans les synaptosomes, RGZS1 et RGSZ2 sont sumoylées à la fois par SUM0-1 et SUMO-2/3. Les formes sumoylées interagissent avec les récepteurs $\mu$ et cette interaction semble impliquée dans la désensibilisation des récepteurs [18]. Ces travaux révèlent un aspect jusqu'alors non suspecté de la sumoylation : la régulation des voies de signalisation des RCPG.

\section{Régulation du trafic axonal des ARNm}

La sumoylation de La, une protéine chaperonne liant les ARN, permet de déterminer la direction du transport des ARN le long de l'axone. La forme sumoylée de La se lie préférentiellement à la dynéine et non à la kinésine comme c'est le cas pour la forme native de La. L'interaction La sumoylée-dynéine permet le transport rétrograde de la protéine La [19].

\section{Régulation de la formation des synapses}

Le facteur de transcription MEF2A (myocyte-specific enhancer factor 2) peut être phosphorylé, acétylé et sumoylé. L'équilibre entre ces modifications posttraductionnelles agit comme un régulateur du développement synaptique. Ainsi, la déphosphorylation de MEF2A induit le passage de la forme MEF2A sumoylée à la forme MEF2A acétylée, ce qui entraîne une inhibition de la formation des synapses [20]. Plus récemment, il a été montré que la protéine kinase CASK $\left(\mathrm{Ca}^{2+} / \mathrm{calmo-}\right.$ dulin (CaM)-activated serine-threonine kinase), impliquée dans la stabilisation des épines dendritiques, est un substrat de la sumoylation [21]. CASK interagit avec plusieurs molécules d'adhésion, dont la protéine 4.1, et cette interaction CASK-4.1 est fortement diminuée 
lorsque CASK est sumoylée. La surexpression d'une forme mimant la sumoylation de CASK (fusion CASK-SUMO) entraîne une diminution de la densité des épines dendritiques ainsi qu'une réduction du volume des épines restantes [21]. La sumoylation intervient par conséquent à la fois dans la genèse et dans la modulation de la morphologie des épines dendritiques.

\section{Rôles de la sumoylation à la membrane plasmique}

\section{Régulation de l'excitabilité neuronale}

L'activité des canaux potassiques de fuite K2Pl dans les neurones a été rapportée comme étant modulée par la sumoylation. En effet, aucun courant n'est enregistré lorsque ces canaux sont exprimés dans l'ovocyte de xénope. La sumoylation de K2Pl pourrait bloquer le pore du canal [22]. Cette étude est toutefois sujette à controverse [23].

\section{Régulation fonctionnelle des récepteurs du glutamate}

Les récepteurs métabotropiques du glutamate du groupe III, qui appartiennent à la famille des RCPG, sont sumoylés [24, 25, 36]. Cependant, le rôle fonctionnel de cette modification et la mise en évidence in vivo de ces récepteurs dans leur état sumoylé restent encore à déterminer.

Dans les neurones de l'hippocampe de rat, la sous-unité GluR6 des récepteurs ionotropiques du glutamate de type kaïnate est un substrat de la sumoylation [17]. La stimulation de ces récepteurs induit leur sumoylation à la membrane plasmique. Les récepteurs sumoylés sont internalisés, ce qui conduit à une modulation de la transmission synaptique dans l'hippocampe (Figure 3). Ce travail représente la première démonstration d'un rôle de la sumoylation au niveau de la synapse et a permis la découverte de fonctions totalement nouvelles de SUMO : son implication dans l'endocytose d'un récepteur et son rôle sur la modulation de la transmission synaptique [17].

\section{Sumoylation des protéines : un rôle dans les maladies neurodégénératives?}

L'accumulation de données sur le rôle de la sumoylation dans les désordres neurologiques constitue une motivation supplémentaire pour mieux comprendre les mécanismes de cette modification des protéines [11]. Les maladies neurodégénératives sont caractérisées par un dysfonctionnement synaptique et une perte progressive des neurones. Cela résulte très fréquemment d'altérations dans la stabilité, la conformation et l'adressage des protéines neuronales. En général, ces anomalies sont la conséquence directe de la dérégulation des modifications post-traductionnelles des protéines et aboutissent à une accumulation cellulaire toxique de protéines sous forme d'inclusions ou d'agrégats. C'est notamment le cas pour une maladie rare du système nerveux central appelée NIID (neuronal intranuclear inclusion disease) (Tableau I). Les patients atteints présentent une ataxie progressive et une démence. Cette maladie est caractérisée par la présence dans les neurones d'inclusions intranucléaires fortement immunoréactives pour SUMO-1 [26]. Cette pathologie pourrait être due à un défaut de désumoylation ou à une action inhibitrice de la sumoylation sur le transport des protéines hors du noyau.

\section{Les maladies par expansion de glutamine}

L'expansion de résidus de glutamine (poly- 0 ) dans certaines protéines conduit à des désordres neurologiques graves. Ces désordres sont caractérisés par des dysfonctions motrices importantes associées en général à des altérations cognitives. Ces maladies regroupent la maladie de Huntington, l'amyotrophie spino-bulbaire (SBMA),

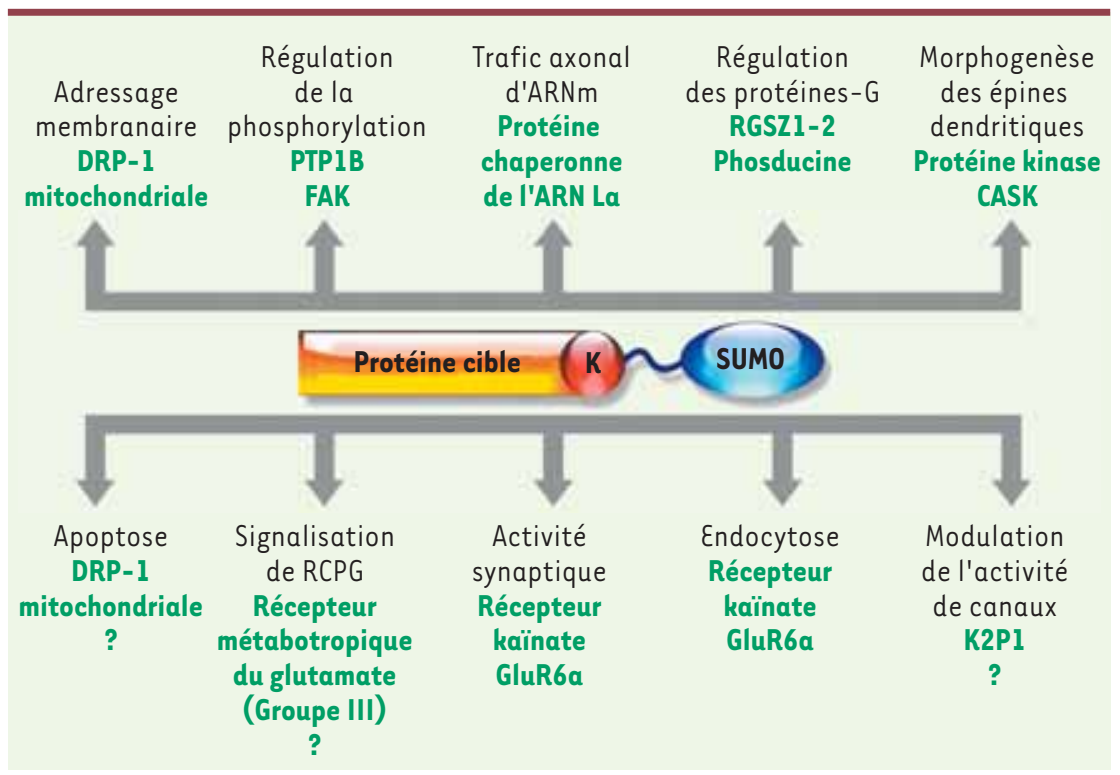

Figure 3. Fonctions extranucléaires de la sumoylation neuronale. Les fonctions non confirmées ou putatives de la sumoylation sont indiquées par un point d'interrogation. CASK, protéine kinase (sérine) dépendante de la calcium/calmoduline; DRP-1, protéine apparentée à la dynamine; FAK, protéine kinase d'adhésion focale; GluR6a, récepteur glutamate $6 a$; PTP1B, protéine tyrosine phosphatase $1 B$; RCPG, récepteur couplé aux protéines-G; RGZS, régulateur de la signalisation des protéines-G. 


\begin{tabular}{|c|c|c|c|c|}
\hline Maladie & $\begin{array}{c}\text { Substrat protéique } \\
\text { sumoylé }\end{array}$ & $\begin{array}{l}\text { Immuno- } \\
\text { réactivité SUMO }\end{array}$ & $\begin{array}{l}\text { Effet de l'expression de SUMO } \\
\text { sur la neurodégénérescence } \\
\text { ou la mort cellulaire }\end{array}$ & Références \\
\hline HD & huntingtine & SUMO-1 +++ & augmenté & [27] \\
\hline SBMA & récepteur des androgènes & SUM0-1 +++ & diminué & [28] \\
\hline DRPLA & atrophine-1 & SUMO-1 +++ & augmenté & [29] \\
\hline SCA de type 1 & ataxine-1 & SUMO-1 +++ & NC & {$[30]$} \\
\hline Maladie NIID & NC & SUMO-1 +++ & augmenté & {$[26]$} \\
\hline Maladie d'Alzheimer & $\begin{array}{c}\text { peptide } \beta \text {-amyloïde } \\
\text { Tau }\end{array}$ & SUMO-3 + & diminué/augmenté & $\begin{array}{c}{[33,34]} \\
{[31]}\end{array}$ \\
\hline Maladie de Parkinson & $\begin{array}{c}\alpha \text {-synucléine } \\
\text { parkine (non covalent) }\end{array}$ & $\begin{array}{l}\text { SUMO-1 + } \\
\text { SUMO-1 + }\end{array}$ & NC & $\begin{array}{l}{[31]} \\
{[32]}\end{array}$ \\
\hline
\end{tabular}

Tableau I. Sumoylation et maladies neurodégénératives. DRPLA : atrophie dentato-rubro-pallido-luysienne; HD : maladie de Huntington; NIID : maladie à inclusions neuronales intranucléaires; SBMA : amyotrophie musculaire spino-bulbaire ; SCA : ataxie cérébro-spinale ; NC : non connu.

l'ataxie cérébro-spinale (SCA) et l'atrophie dentato-rubro-pallidoluysienne (DRPLA). Les tissus cérébraux provenant de patients atteints de ces maladies présentent tous des inclusions intranucléaires immunoréactives pour SUMO-1 (Tableau I).

La maladie de Huntington résulte de l'expansion de poly- $\varrho$ dans la protéine huntingtine. Chez la drosophile, une lysine de cette protéine est sumoylée, conduisant à une inhibition de son ubiquitinylation [37]. La conséquence directe est une inhibition de la dégradation de la protéine et une augmentation intracytoplasmique de la concentration du fragment neurotoxique. De plus, la réduction de l'expression de SUMO-1 diminue la dégénérescence neuronale dans ce modèle génétique de maladie de Huntington [27]. Inversement, l'inhibition de la voie de sumoylation augmente de manière significative la neurodégénérescence dans un modèle de SBMA [28]. II est par conséquent important de constater qu'un équilibre entre ubiquitinylation et sumoylation est essentiel pour le contrôle à la fois de la stabilité et l'adressage cellulaire de ces protéines et que cette balance est dérégulée dans ces deux maladies.

La DRPLA résulte d'une expansion de poly- 0 dans l'atrophine- 1 , une protéine de fonction inconnue. La surexpression de SUMO-1 dans une lignée neuronale conduit à une augmentation importante du nombre d'inclusions intranucléaires, suivie d'une exacerbation de la mort cellulaire [29].

La SCA de type 1 correspond à une expansion de poly- $\rho$ dans l'ataxine1. L'ataxine- 1 est sumoylée sur 5 résidus de lysines et l'addition de poly- $\rho$ réduit le niveau de sumoylation de la protéine [30]. Cependant, les conséquences fonctionnelles de cette diminution des niveaux de sumoylation restent à déterminer.

\section{La maladie de Parkinson}

Cette maladie est caractérisée par une perte importante de neurones dopaminergiques dans la substance noire avec accumulation d'inclusions appelées corps de Lewy. Ces inclusions sont immunoréactives pour SUM0-1 et pour l' $\alpha$-synucléine, un substrat de la sumoylation [31].
L'existence de la sumoylation de l' $\alpha$-synucléine native ainsi que la fonction de cette modification n'ont cependant pas été établies.

La parkine est une ubiquitine ligase impliquée dans le parkinsonisme juvénile autosomique récessif [38]. Elle interagit de façon non covalente avec SUMO-l et cette interaction conduit à une augmentation de son activité de ligase de l'ubiquitine [32]. Ceci représente un exemple intéressant de régulation de la voie de l'ubiquitinylation par la voie de sumoylation par le biais d'une interaction non covalente.

\section{La maladie d'Alzheimer}

Cette maladie est caractérisée par la formation neurotoxique de plaques riches en peptide amyloïde $(A \beta)$ et par l'agrégation de la protéine Tau sous la forme de filaments neurofibrillaires pathologiques. Tau est un substrat de la sumoylation et est également ubiquitinylée. L'inhibition de la dégradation des protéines par le protéasome augmente I'ubiquitinylation de Tau et diminue sa sumoylation [31], révélant l'existence d'une régulation fine entre ces deux modifications post-traductionnelles pour le maintien de la stabilité de Tau. Bien que les résultats soient controversés [33], la surexpression de SUMO-3 diminue dramatiquement la production du peptide $A \beta$ impliquant ainsi directement la sumoylation dans la maladie d'Alzheimer [34].

\section{Conclusions}

De nombreux progrès ont récemment été réalisés qui améliorent notre compréhension de la fonction de la sumoylation dans le système nerveux central et de son 
implication dans les maladies neurodégénératives. La sumoylation participe à la régulation de nombreuses voies de signalisation cellulaire et la dérégulation des mécanismes conduisant à la sumoylation des protéines a sans nul doute des conséquences importantes pour les fonctions neuronales. II existe un grand nombre de protéines sumoylées au niveau de la synapse [17] mais leur nature et leur fonction restent à ce jour inconnues. L'identification de la fonction des substrats synaptiques de la sumoylation peut permettre de mieux comprendre le rôle joué par la sumoylation dans la régulation des fonctions synaptiques et par conséquent dans la physiologie du neurone. L'un des défis majeurs sera de déterminer si des dysfonctionnements dans la régulation ou la localisation des activités enzymatiques de la sumoylation peuvent avoir un impact sur la mise en place ou l'aggravation des symptômes des maladies neurodégénératives humaines. $\diamond$

\section{SUMMARY}

\section{Extranuclear functions}

of protein sumoylation in the central nervous system

Post-translational protein modifications play essential roles in many aspects of cellular functions and therefore in the maintenance of cell integrity. These protein modifications are involved at all stages of neuronal communication within the central nervous system. Sumoylation is a reversible post-translational protein modification that consists in the covalent labelling of a small protein called SUMO to lysine residues of selected target proteins. Sumoylation is a well characterized regulator of nuclear functions and has recently emerged as a key factor for numerous extranuclear processes. Furthermore, sumoylation has recently been shown to modulate synaptic transmission and is also implicated in a wide range of neurodegenerative diseases. $\diamond$

\section{REMERCIEMENTS}

S. Martin bénéficie d'un contrat ATIP «neurobiologie» du Centre National de la Recherche Scientifique.

\section{CONFLIT D'INTÉRÊTS}

L'auteur déclare n'avoir aucun conflit d'intérêts concernant les données publiées dans cet article.

\section{RÉFÉRENCES}

1. Matunis MJ, Coutavas $\varepsilon$, Blobel G. A novel ubiquitin-like modification modulates the partitioning of the Ran-GTPase-activating protein RanGAPI between the cytosol and the nuclear pore complex. J Cell Biol 1996; 135 : 1457-70.

2. Hayashi T, Seki M, Maeda D, et al. Ubc9 is essential for viability of higher eukaryotic cells. Exp Cell Res $2002 ; 280: 212-21$.

3. Nacerddine K, Lehembre F, Bhaumik M, et al. The SUMO pathway is essential for nuclear integrity and chromosome segregation in mice. Dev Cell $2005 ; 9: 769-79$.

4. Tatham MH, Jaffray $\varepsilon$, Vaughan $O A$, et al. Polymeric chains of SUMO-2 and SUMO-3 are conjugated to protein substrates by SAE1/SAE2 and Ubc9. J Biol Chem $2001 ; 276: 35368-74$.

5. Hay RT. SUMO: a history of modification. Mol Cell 2005 ; 18 : 1-12.

6. Mukhopadhyay D, Dasso M. Modification in reverse: the SUMO proteases. Trends Biochem Sci 2007 ; $32: 286-95$.

7. Geiss-Friedlander R, Melchior F. Concepts in sumoylation: a decade on. Nat Rev Mol Cell Biol 2007 ; $8: 947-56$.

8. Heun P. SUMOrganization of the nucleus. Curr Opin Cell Biol $2007 ; 19: 350-5$.

9. Perry JJ, Tainer JA, Bobby MN. A SIM-ultaneous role for SUMO and ubiquitin. Trends Biochem Sci 2008 ; $33: 201-8$.

10. Kerscher 0 . SUMO junction-what's your function? New insights through SUMO-interacting motifs. $E M B O$ Rep $2007 ; 8: 550-5$.

11. Martin S, Wilkinson KA, Nishimune A, Henley JM. Emerging extranuclear roles of protein SUMOylation in neuronal function and dysfunction. Nat Rev Neurosci $2007 ; 8$ : 948-59.
12. Kadare G, Toutant M, Formstecher $\varepsilon$, et al. PIAS1-mediated sumoylation of focal adhesion kinase activates its autophosphorylation. J Biol Chem 2003; $278: 47434-40$.

13. Dadke S, Cotteret $S$, Yip SC, et al. Regulation of protein tyrosine phosphatase 1 B by sumoylation. Nat Cell Biol $2007 ; 9: 80-5$.

14. Harder Z, Zunino R, McBride H. Sumol conjugates mitochondrial substrates and participates in mitochondrial fission. Curr Biol $2004 ; 14: 340-5$.

15. Wasiak S, Zunino R, McBride HM. Bax/Bak promote sumoylation of DRPl and its stable association with mitochondria during apoptotic cell death. J Cell Biol 2007 ; 177 : 439-50.

16. Zunino R, Schauss A, Rippstein P, et al. The SUMO protease SENP5 is required to maintain mitochondrial morphology and function. J Cell Sci $2007 ; 120: 1178-88$.

17. Martin S, Nishimune A, Mellor JR, Henley JM. SUMOylation regulates kainatereceptor-mediated synaptic transmission. Nature 2007 ; 447 : 321-5.

18. Rodriguez-Munoz M, Bermudez D, Sanchez-Blazquez P, Garzon J. Sumoylated RGS-Rz proteins act as scaffolds for Mu-opioid receptors and G-protein complexes in mouse brain. Neuropsychopharmacology $2007 ; 32: 842-50$.

19. Van Niekerk $E A$, Willis $D E$, Chang JH, et al. Sumoylation in axons triggers retrograde transport of the RNA-binding protein La. Proc Natl Acad Sci USA $2007 ; 104: 12913-8$.

20. Shalizi A, Gaudilliere B, Yuan Z, et al. A calcium-regulated MEF2 sumoylation switch controls postsynaptic differentiation. Science 2006 ; 311 : 1012-7.

21. Chao HW, Hong CJ, Huang TN, et al. SUMOylation of the MAGUK protein CASK regulates dendritic spinogenesis. J Cell Biol 2008; 182 : 141-55.

22. Rajan S, Plant LD, Rabin ML, et al. Sumoylation silences the plasma membrane leak $\mathrm{K}^{+}$channel K2P1. Cell $2005 ; 121$ : 37-47.

23. Feliciangeli S, Bendahhou S, Sandoz G, et al. Does Sumoylation control K2P1/TWIKl background K+ channels? Cell 2007 ; 130 : 563-69.

24. Tang Z, \&I Far 0 , Betz H, Scheschonka A. Piasl interaction and sumoylation of metabotropic glutamate receptor 8. J Biol Chem 2005; 280 : 38153-9.

25. Wilkinson KA, Nishimune A, Henley JM. Analysis of SUMO-1 modification of neuronal proteins containing consensus SUMOylation motifs. Neurosci Lett $2008 ; 436: 239-44$.

26. Pountney DL, Huang $Y$, Burns RJ, et al. SUMO-1 marks the nuclear inclusions in familial neuronal intranuclear inclusion disease. Exp Neurol 2003; $184: 436-46$.

27. Steffan JS, Agrawal N, Pallos J, et al. SUMO modification of Huntingtin and Huntington's disease pathology. Science 2004 ; 304 : 100-4.

28. Chan HY, Warrick JM, Andriola I, et al. Genetic modulation of polyglutamine toxicity by protein conjugation pathways in Drosophila. Hum Mol Genet $2002 ; 11: 2895-904$

29. Terashima T, Kawai H, Fujitani M, et al. SUMO-1 co-localized with mutant atrophin-1 with expanded polyglutamines accelerates intranuclear aggregation and cell death. Neuroreport $2002 ; 13: 2359-64$.

30. Ueda H, Goto J, Hashida H, et al. Enhanced SUMOylation in polyglutamine diseases. Biochem Biophys Res Commun 2002 ; 293 : 307-13.

31. Dorval V, Fraser PE. Small ubiquitin-like modifier (SUMO) modification of natively unfolded proteins tau and alpha-synuclein. J Biol Chem 2006 ; $281: 9919-24$.

32. Um JW, Chung KC. Functional modulation of parkin through physical interaction with SUMO-1. J Neurosci Research 2006 ; 84 : 1543-54.

33. Dorval V, Mazzella MJ, Mathews PM, et al. Modulation of Abeta generation by small ubiquitin-like modifiers does not require conjugation to target proteins. Biochem J $2007 ; 404$ : 309-16.

34. Li Y, Wang H, Wang S, et al. Positive and negative regulation of APP amyloidogenesis by sumoylation. Proc Natl Acad Sci USA 2003; 100 : 259-64.

35. Buyse M, Aparicio T, Guilmeau S, et al. Paracrine actions of the stomachderived leptin. Med Sci (Paris) $2004 ; 20: 183-8$.

36. Galvez T, Pin JP. Comment fonctionne un récepteur couplé aux protéines $G$ ? Le cas des récepteurs des neurotransmetteurs métabotropiques du glutamate et du GABA. Med Sci (Paris) 2003; 19 : 559-65.

37. Liévens JC, Birman S. La chorée de Huntington chez la drosophile et chez la souris: vers de nouvelles pistes thérapeutiques?. Med Sci (Paris) 2003; $19: 593-9$.

38. Corti 0 , Brice $A$. La maladie de Parkinson: que nous apprennent les gènes responsables des formes familiales? Med Sci (Paris) 2003 ; 19 : 613-9.
TIRÉS À PART

S. Martin 